\title{
Observations of nonthermal plasmas at different aspect angles
}

Article

Published Version

Winser, K. J., Lockwood, M., Jones, G. O. L. and Suvanto, K. (1989) Observations of nonthermal plasmas at different aspect angles. Journal of Geophysical Research, 94 (A2). pp. 14391449. ISSN 0148-0227 doi:

https://doi.org/10.1029/JA094iA02p01439 Available at https://centaur.reading.ac.uk/38885/

It is advisable to refer to the publisher's version if you intend to cite from the work. See Guidance on citing.

Published version at: http://dx.doi.org/10.1029/JA094iA02p01439

To link to this article DOI: http://dx.doi.org/10.1029/JA094iA02p01439

Publisher: American Geophysical Union

All outputs in CentAUR are protected by Intellectual Property Rights law, including copyright law. Copyright and IPR is retained by the creators or other copyright holders. Terms and conditions for use of this material are defined in the End User Agreement.

\section{www.reading.ac.uk/centaur}

\section{CentAUR}

Central Archive at the University of Reading 
Reading's research outputs online 


\title{
OBSERVATIONS OF NONTHERMAL PIASMAS AT DIFFERENT ASPECT ANGLES
}

\author{
K. J. Winser and M. Iockwood
}

Rutherford Appleton Iaboratory, Chilton, Didcot, Oxon, England

G. O. I. Jones

University College of Wales, Aberystwyth, Dyfed

K. Suvanto

Blackett Iaboratory, Inperial College, Iondon

Abstract. Data are presented from the EISCAT (European Incoherent Scatter (Facility)) CP-3-E experiment which show large increases in the auroral zone convection velocities ( $>2 \mathrm{~km} \mathrm{~s}^{-1}$ ) over a wide range of latitudes. These are larger than the estimated neutral thermal speed and allow a study of the plasma in a nonthermal state over a range of observing angles. Spectra are presented which show a well-defined central peak, consistent with an ion velocity distribution function which significantly departs from a Maxwellian form. As the aspect angle decreases, the central peak becomes less obviolis. Simulated spectra, derived using theoretical expressions for the $\mathrm{O}^{+}$ion velocity distribution function based on the generalized relaxation collision model, are compared with the observations and show good first-order, qualitative agreement. It is shown that ion temperatures derived from the observations, with the assumption of a Maxwellian distribution function, are an overestimate of the true ion temperature at large aspect angles and an underestimate at low aspect angles. The theoretical distribution functions have been included in the "standard" incoherent scatter radar analysis procedure, and attempts have been made to derive realistic ionospheric parameters from nonthermal plasma observations. If the expressions for the distribution function are extended to include mixed ion composition, a significant improvement is found in fitting some of the observed spectra, and estimates of the ion composition can be made. The non-Maxwellian analysis of the data revealed that the spectral shape distortion parameter, $D^{\star}$, was significantly higher in this case for molecular ions than for atomic ions in a thin height slab roughly $40 \mathrm{~km}$ thick. This would seem unlikely if the main molecular ions present were $\mathrm{NO}^{*}$. We therefore suggest that $\mathrm{N}^{*}$ formed a significant proportion of the molecular ions present during these observations.

\section{Introduction}

The role of ion-neutral collisions was first introduced into the study of high-latitude ionospheric ion-velocity distributions by Schunk

Copyright 1989 by the American Geophysical Union.

Paper number 88JA03289

0148-0227/89/88JA-03289\$05.00 and Walker [1972]. Their results showed that in the presence of such collisions the ion-velocity distribution function would become anisotropic and significantly distorted from a Maxwellian form (usually associated with thermal equilibrium) when subjected to dc electric fields of the type observed frequently in the auroral regions.

For moderate electric fields the distribution function can be reasonably represented by a biMaxwellian, which can be described in terms of the perpendicular and parallel (with respect to B) ion temperatures, $T_{\perp}$ and $T_{i l}$. St-Maurice and Schunk [1973, 1974] evaluated distribution functions for the simple relaxation collision model and found that at heights where the ionneutral collision frequency is considerably less than the ion gyrofrequency (typically above about $160 \mathrm{~km}$ ), the $\mathrm{O}^{7}$ velocity distribution will become toroidal when the electric field exceeds about 40 $\mathrm{mV} \mathrm{m}^{-1}$. However, it is now widely accepted that the relaxation model is a very simplistic picture of collision processes in the high-latitude ionosphere. Barakat et al. [1983] used a MonteCarlo technique to evaluate the $\mathrm{O}^{+}$velocity distribution, for a more realistic collision model (i.e., a mixture of resonant charge exchange and polarization scatter interactions). They showed that toroidal distribution functions could indeed form, but only for much larger electric fields than had been predicted before (typically > $80 \mathrm{mV} \mathrm{m}^{-1}$ ).

St-Maurice et al. [1976] made observations of non-Maxwellian distribution functions using a Retarding Potential Analyzer (RPA) on board the $\mathrm{AE}-\mathrm{C}$ satellite. These, however, were only of limited value for three reasons: First, information was only obtained in the fieldperpendicular plane; second, it was not possible to resolve $\mathrm{O}^{+}$and $\mathrm{NO}^{+}$ions when the ion temperature was very large (resulting from local frictional heating), and hence the RPA studies could not include the more highly distorted distribution functions; and third, the spatialtemporal ambiguity problem of satellite data did not allow studies of the temporal evolution of the nonthermal plasma.

Perraut et al. [1984] described tristatic EISCAT (European Incoherent Scatter (Facility)) incoherent scatter radar observations which showed strong evidence for a bi-Maxwellian velocity distribution and a clear temperature anisotropy. Iovhaug and Fla [1986] later presented temperature anisotropies which on occasion reached values which implied non- 
Maxwellian ion velocity distributions. Raman et al. [1981] predicted the effect of the nonMaxwellian distribution function used by StMaurice et al. [1976] on the spectra of incoherent scatter signals. Hubert [1983, 1984] has presented a refinement of the analytic method used and similarly predicted the incoherent scatter spectra. The first ground-based observations of non-Maxwellian plasma were presented by Lockwood et al. [1987] using data from the EISCAT observing program UK-POIAR, where the angle $(\Psi)$ between the direction of the radar beam and geomagnetic field remained large and fixed (roughly $73.5^{\circ}$ ). This work showed the spectral forms predicted by Raman et al. [1981] and Hubert [1984] and that nonthermal distributions could last for periods of at least several minutes. Winser et al. [1987] presented further results from the EISCAT radar which showed that nonthermal plasma could exist in a quasi-steady state (for periods up to 1 hour) and how the observation of such plasma is very sensitive to aspect angle. They also showed that the interpretation of such data with the assumption of an isotropic Maxwellian distribution function leads to erroneous estimates of the plasma temperatures as predicted by Raman et al. [1981] and later confirmed by Hubert [1984]. Other recent observations of nonthermal plasma by EISCAT have been reported by Moorcroft and Schlegel [1988] and Lockwood et al. [1988, also Incoherent scatter radar observations of non-Maxwellian ion velocity distributions in the auroral F region, submitted to Journal of Geophysical Research, 1988].

In this paper we attempt to analyze the data presented by Winser et al. [1987] using the theoretical distribution function described by Raman et al. This type of fit has been carried out previously by Moorcroft and Schlegel [1988]; however, their observed spectra were very noisy, and their analysis suffered severely because of this. A comparison between the results presented here from the non-Maxwellian analysis and those deduced from an analysis which assumes an isotropic Maxwellian distribution function, shows that the derived "Maxwellian" ion temperature, $\mathrm{T}_{\mathrm{im}}$, is an overestimate of the true ion temperature at large aspect angles but is underestimated for low aspect angles. We have also extended this work by including the effects of mixed ion composition in our analysis, which has not previously been attempted before. This shows that on occasions a significant improvement can be found in fitting the observed spectra when allowances are made for the presence of molecular ions. Finally, we show that the distribution function described by Raman et al. adequately describes the true distribution, at least to a first order, for aspect angles between about 35 and $75^{\circ}$.

In section 2 we give an overview of the data discussed in this paper and briefly describe the procedure for fitting non-Maxwellian spectra. Section 3 describes the initial results of trying to fit the observed non-Maxwellian data with the assumption of a pure $0^{+}$ionosphere. In section 4 we discuss the effects of a mixed composition on the theoretical spectra and the problems this introduces in the data analysis. Finally, we summarise our findings in section 5 .

\section{Observations}

Winser et al. [1987] described experimental data which showed the existence of non-thermal plasma for an extended period ( $\approx 1$ hour) in a time sector consistent with the model predictions of Lockwood and Fuller-Rowell [1987a, b] following a sudden increase in cross-cap potential. The observations were made on August 27, 1986, using the EISCAT common program CP-3-E and will form the basis of the work described here. CP-3-E is a latitude scanning program where the beam scans in a plane approximately perpendicular to the magnetic I shells, with a cycle time of $30 \mathrm{~min}$. The 17-position cycle allowed measurements of plasma parameters over a range of viewing angles with respect to the geomagnetic field $(\Psi)$ from 0 to $73 \mathrm{deg}$ and invariant latitudes, $\Lambda$, from 61 to $72.5 \mathrm{deg}$ at a height of $275 \mathrm{~km}$. The beams of the remote receiving stations at Kiruna and Sodankyla intersect the beam of the transmit/receive antenna (located at Tromso) at $275 \mathrm{~km}$ for all 17 positions. This gave direct measurements of three independent components of the bulk ion drift and hence allowed the instantaneous perpendicular electric field vector to be determined without the temporal and spatial homogeneity assumption inherent in the commonly used "beam-swinging" technique.

The period of interest is that between 1300 and 1400 UT where the electric field was significantly enhanced, yielding ion velocities as large as $2.5 \mathrm{~km} \mathrm{~s}^{-1}$ over $5 \mathrm{deg}$ of latitude at the beam intersection height (refer to Figure 2 of Winser et al. [1987]).

From simple theoretical considerations it is found useful to define a parameter, $D^{\prime}$, as the ratio of the relative ion-neutral drift velocity and the neutral thermal speed, or

$$
D^{\prime}=\frac{\left|\underline{V}_{p}-\underline{v}_{n}\right|}{\sqrt{\left(2 k T T_{n} / m_{n}\right)}}=\frac{\left|\underline{v}_{p}-\underline{v}_{n}\right|}{V_{t h}}
$$

where $V_{p}$ and $V_{n}$ are the plasma and neutral velocities respectively, $\mathrm{T}_{\mathrm{n}}$ is the neutral temperature, $m_{n}$ is the neutral mass, $k$ is Boltzmann's constant, and $v_{t h}$ is the neutral thermal speed.

The values of $D^{\prime}$ for which non-Maxwellian or toroidal plasmas may be observed are not yet fully known. Departures from a bi-Maxwellian are apparent for the relaxation model when $D^{\prime}>0.75$, however, Barakat et al. [1983] concluded that toroidal distributions would only form for much larger ion drifts, typically $D^{\prime}>1.5$. We should point out however, that the formation of a nonMaxwellian velocity distribution is not a threshold effect: Departures from a Maxwellian form will occur in the presence of any electric fields for the simple relaxation model case.

It was possible to estimate the neutral thermal speed for this period with the assumption that the neutral temperature was equal to the ion temperature for the periods immediately before and after, when the electric fields were small and steady both in magnitude and direction. This can be further justified since the value derived agreed well with that obtained from a global, time-dependent, three-dimensional coupled 
ionosphere-thermosphere model (T. J. FullerRowell et al., Interactions between neutral thermospheric composition and the polar ionosphere using a coupled ionospherethermosphere model, submitted to Journal of Geophysical Research, 1988) run under similar conditions to those prevailing at the time of these observations [Lockwood and Fuller-Rowell, $1987 \mathrm{a}, \mathrm{b}] . \mathrm{V}_{\text {th }}$ was estimated to be $1.04 \pm 0.05 \mathrm{~km}$ $\mathrm{s}^{-1}$ (for $\mathrm{T}_{n}=1050 \pm 100 \mathrm{~K}$ ). This is considerably smaller than the magnitudes of the observed ion flows, and so this day provides an almost perfect data set to study nonthermal plasma over a large range of aspect angles.

Winser et al. [1987] concluded that the ion velocity distribution function for this period was non-Maxwellian and possibly toroidal. This was based on (1) the shape of the observed spectra, (2) the observed ion temperature anisotropy, and (3) the estimated values of $D^{\prime}$. We now wish to extend this work by showing the results of fitting the observed nonthermal spectra using a variant of the algorithm developed by Raman et al. [1981] to estimate the velocity distribution function for the "generalized relaxation model", as has recently been done by Moorcroft and Schlegel [1988] and Suvanto et al. (Analysis of incoherent scatter radar data from non-Maxwellian F region plasma, submitted to Journal of Atmospheric and Terrestrial Physics, 1988) (hereinafter referred to as "submitted"). The algorithm has been improved in that lengthy integrations are now performed analytically rather than numerically [Suvanto, 1988], which reduces the computation time and is more suited to fitting large numbers of observed spectra.

\subsection{Fitting non-Maxwellian Spectra}

The average (three-dimensional) temperature, $\mathrm{T}_{i}$ (defined from the mean square of the speed of the random ion velocity), can be expressed in terms of the one-dimensional temperatures $T_{11}$ and $\mathrm{T}_{\perp}$ (defined from the mean square of the parallel and perpendicular velocity components respectively) as follows:

$$
T_{i}=\left(T_{\|}+2 T_{\perp}\right) / 3
$$

Similarly, a one-dimensional line-of-sight temperature, $\mathrm{T}_{\mathrm{f}}$, can be defined for any given direction at an angle $\Psi$ to the geomagnetic field using the expression

$$
\mathrm{T}_{\mathrm{f}}=\mathrm{T}_{\|} \cos ^{2} \Psi+\mathrm{T}_{\perp \sin ^{2} \Psi}
$$

We have adopted the definitions of temperature (both one- and three-dimensional) as given by Raman et al. [1981] and Moorcroft and Schlegel [1988]. In circumstances where the plasma is isotropic then $\mathrm{T}_{\perp}, \mathrm{T}_{\|}, \mathrm{T}_{\mathrm{i}}$ and $\mathrm{T}_{\mathrm{f}}$ will all be equal; however, in the presence of large electric fields these can be different by up to several hundred degrees [Raman et al., 1981]. From (1) and (2) it can be seen that $T_{i}=T_{f}$ for $\Psi=$ $54.7^{\circ}$.

For sufficiently large electric fields the ion velocity distribution is expected to become nonMaxwellian. (The mechanisms which result in such distributions are discussed in some detail by StMaurice and Schunk [1973, 1974, 1979] and, more recently by M. Lockwood et al. (Incoherent scatter radar observations of non-Maxwellian ion velocity distributions in the auroral $F$ region, submitted to Journal of Geophysical Research, 1988) and will not be discussed any further here.) The expression for the distribution function can then be expanded to include the adjustable parameters $D^{*}$ and $T^{*}$, the values of which have to be determined empirically. $D^{*}$ is described as a shape distortion parameter and is equal to zero for a Maxwellian distribution function and approximately 1.2 for an $0^{+}$torus in the field perpendicular direction. $\mathrm{T}^{*}$ determines the width of the distribution and acts in a similar way to $\mathrm{T}_{i}$ in a Maxwellian case. $\mathrm{T}^{*}=\mathrm{T}_{n}$ for a pure relaxation collision process but can also be expressed in terms of $\mathrm{T}_{i}$ and $\mathrm{D}^{*}$ as follows:

$$
\mathrm{T}^{*}=\mathrm{T}_{\mathrm{i}} /\left(1+2 / \mathrm{D}^{* 2}\right)
$$

The parallel and perpendicular temperatures for the distribution function given by Raman et al. [1981] are

$$
\begin{aligned}
& \mathrm{T}_{\|}=\mathrm{T}^{*} \\
& \mathrm{~T}_{\perp}=\mathrm{T}^{*}\left(1+\mathrm{D}^{\star 2}\right) \\
& \mathrm{T}_{\mathrm{f}}=\mathrm{T}^{*}\left(1+\mathrm{D}^{\star 2} \sin ^{2} \Psi\right)
\end{aligned}
$$

The current standard method of analyzing incoherent scatter radar data is to compare the observed spectra with theoretical spectra derived using isotropic Maxwellian velocity distributions and deduce values for electron density and electron and ion temperature using a least squares fitting technique. The distribution function was replaced by that for the generalized relaxation model described by Raman et al. [1981], calculated using the semianalytic approach of Suvanto [1988]. We have introduced a fourth parameter, $D^{*}$, in the fitting procedure and in the first instance assumed that the ionosphere at $275 \mathrm{~km}$ consisted solely of $\mathrm{O}^{+}$ions. The analysis program was then run, fitting for $\mathrm{N}_{e}, \mathrm{~T}_{e}, \mathrm{D}^{*}$, and $\mathrm{T}_{i}$ (which incidentally gave the same answers as fitting for $\mathrm{N}_{e}, \mathrm{~T}_{e}, \mathrm{D}^{*}$, and $\mathrm{T}_{f}$ ). Details of the implementation of the Suvanto [1988] spectrum synthesis routines in our IS data analysis program have been given by Suvanto et al. (submitted, 1988).

\section{Parameters Fitted From Non-Maxwellian Spectra}

Figure 1 shows the observed incoherent scatter spectra from Tromso (at $275 \mathrm{~km}$ ) for positions 3 to 10 (covering a range of aspect angles from 0 to $71^{\circ}$ ) in the scan period 1300 to 1330 UT. These are reproduced from Figure 3 of Winser et al. [1987] and have been postintegrated for the dwell period of the radar in each position (typically $100 \mathrm{~s})$. Because of the nature of the data gathering process it is not possible to put error bars on the measured spectra; however, a careful study of the individual spectra at the basic time resolution of $10 \mathrm{~s}$ revealed precisely the same features that are presented here but with stochastic noise added. Reference to Figure 2 of Winser et al. [1987] will show that the measured bulk ion drift at positions 3 and 4 was not 


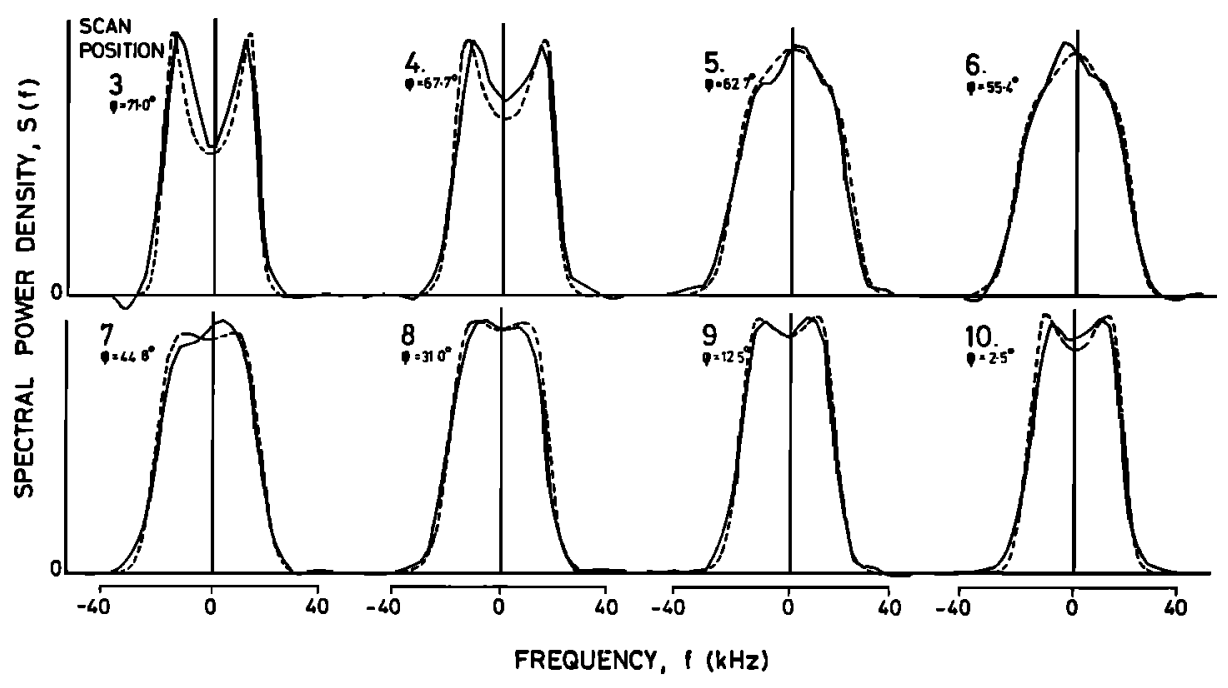

Fig. 1. Incoherent scatter spectra observed at altitude $275 \mathrm{~km}$ in positions 3 to 10 of the EISCAT experiment mode CP-3-E between 1300 and 1330 UT on August 27, 1986. Superimposed on these are the best fit, theoretical, non-Maxwellian spectra with the assumption of a single-species $\left(0^{+}\right)$ionosphere (broken lines).

unusually large, and it is clear that the observed spectra are characteristic of Maxwellian velocity distributions. On the other hand, the convection velocities measured at positions 5 to 10 were considerably enhanced $\left(\approx 2 \mathrm{~km} \mathrm{~s}^{-1}\right)$. The corresponding spectra show a well-defined central peak at the greatest aspect angle (position 5), which gradually decreases for decreasing $\psi$.

Superimposed on the observed spectra are the theoretical "fitted" spectra derived using the analysis procedure described above. Clearly, there is very good qualitative agreement between the measured and synthesized spectra, with all the main features reproduced. These give better (lower variance) fits to the observed data (and hence plasma parameters are more accurately

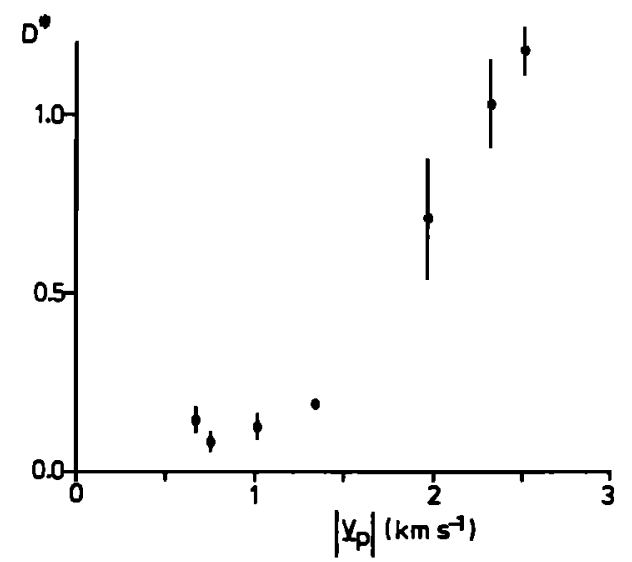

Fig. 2. Fitted $D^{*}$ values as a function of the bulk plasma drift using data obtained between 1300 and 1315 UT on August 27, 1986. This plot is made up only from data which were measured at aspect angles greater than $30 \mathrm{deg}$. derived) than would be obtained by fitting the measured spectra with synthesized spectra which assume a Maxwellian line-of-sight ion velocity distribution. There are, however, some very important features of the observed spectra which synthesized spectra for pure $\mathrm{O}^{+}$plasma cannot be made to reproduce, most notably the well defined "shoulders" clearly observed for position 5 in the scan. (Refer to section 4 for a more detailed discussion on this point.)

Figure 2 shows fitted values of $D^{*}$ as a function of the bulk ion drift speed $\left(V_{p}\right)$ using data obtained during the period 1300 to $1330 \mathrm{UT}$. $D^{*}$ was derived using the analysis procedure described above, which included the distribution function for $\mathrm{O}^{+}$ions as described by Raman et al (1981). As D' increases above about 1.0 (estimated to be near $V_{p}=1 \mathrm{~km} \mathrm{~s}^{-1}$ ), $D^{*}$ steadily rises, reaching values around 1.1 for $V_{p}=2.5 \mathrm{~km}$ $\mathrm{s}^{-1}$. This clearly indicates the nonthermal nature of the plasma when subjected to enhanced electric fields. It should be pointed out that this plot is made up only from data taken at aspect angles larger than $30 \mathrm{deg}$. This limit arises because the expression for the distribution function cannot be used for look directions that are fieldaligned or close to it [Raman et al., 1981], and conseguently, at lower aspect angles, attempts to fit $D^{*}$ are meaningless (see below, and Suvanto et al. (submitted, 1988)). Note that the scan positions far to the north, where the aspect angle is large but the ion drift was low [see Winser et al., 1987], gave low $D^{*}$ values, with low errors. This is a little surprising from visual inspection of synthesized spectra, as variation of the spectra over the range $0<D^{*}<0.5$ is difficult to discern. However, the least squares analysis of these data is showing that, statistically, a significantly worse fit is obtained if $\mathrm{D}^{*}$ is increased above the maximum of the error bar, hence we believe that these data points for low ion drift are valid. This point is discussed further by Suvanto et al. (submitted, 1988). 


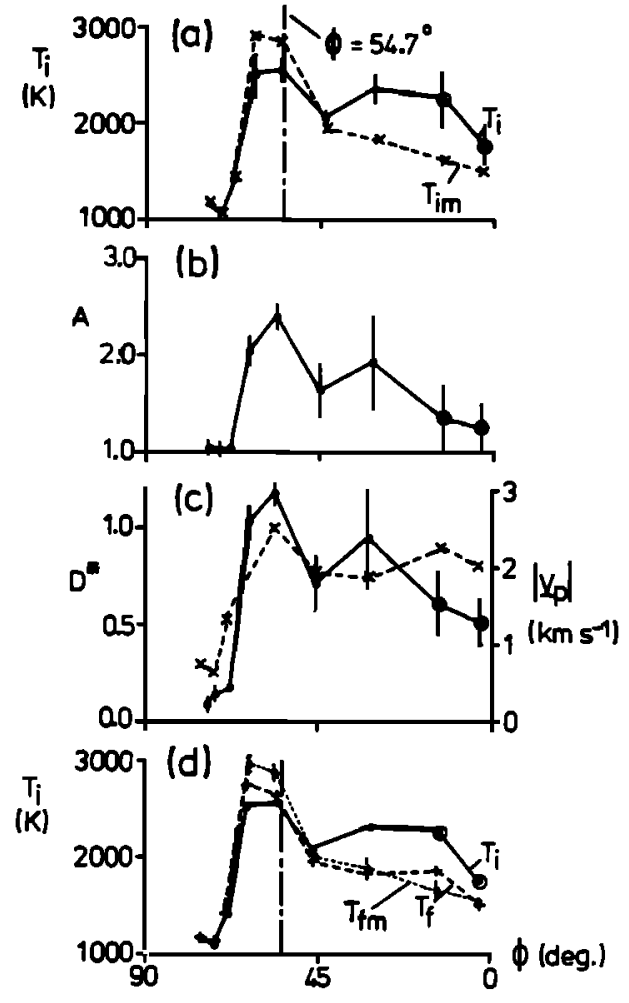

Fig. 3. Plasma parameters at $275 \mathrm{~km}$ altitude as a function of aspect angle $(\Psi)$. These were derived from spectra observed in the period 1300 to 1330 UT on August 27. (a) Comparison of the ion temperature derived from a single-species non-Maxwellian analysis $\left(T_{i}\right)$ with that derived from, the data using the standard analysis method, which assumes an isotropic Maxwellian velocity distribution (Tim). (b) The temperature anisotropy, $A\left(=T_{\perp} / \mathbb{T}_{\|}\right)$. (c) Comparison of the fitted. $D^{\text {i }}$ values from a non-Maxwellian analysis (solid line) with the bulk plasma drift (broken line) measured in each position. (d) Comparison of $\mathrm{T}_{j}$ (same as in Figure $3 \mathrm{a}$ ) with the line-of sight temperatures, $\mathrm{T}_{\mathrm{f}}$ and $\mathrm{T}_{\mathrm{fm}}$, derived from the non-Maxwellian fits (using equation (2)) and the standard analysis program, which assumes an isotropic Maxwellian distribution, respectively. The points encompassed with open circles on the plots for $T_{i}, A$ and $D^{*}$ should be disregarded, as the distribution function used in the analysis is insensitive to deviations from a Maxwellian in or close to the field-parallel direction.

Figure 3 summarizes plasma parameters derived from non-Maxwellian fits to the data as a function of aspect angle, $\Psi$. The temperature anisotropy factor, $A\left(=T_{\perp} / T_{\|}\right)$, is plotted in Figure $3 \mathrm{~b}$. This was obtained using (3), (4), and (5) and shows values as high as 2.3 for the largest observed convection flows. It should be noted that this is not based on measurements of $T_{\perp}$ and $T_{\|}$but is obtained from the fitted values of $D^{*}$ and hence assumes the generalized form of the relaxation model distribution function adopted by Raman et al. The validity of these anisotropy estimates depends on the validity of the adopted distribution function and will be discussed later. Figure $3 \mathrm{c}$ shows tine bulk ion drift magnitude (dashed line) and the fitted $D^{*}$ values (solid line) as a function of $\Psi$. This illustrates several points which are not obvious from Figure 2: First, the convection velocities were not significantly enhanced in the northernmost positions of the scan (refer to Figure 2 of Winser et al., [1987]), and the observed spectra in these positions were characteristic of a plasma in thermal equilibrium. It is important to note that the non-Maxwellian fits to these spectra produce $D^{\star}$ values that are low $(\approx 0.1)$. Second, the values of $D^{*}$ fitted for aspect angles less than about 30 deg (two values of 0.61 and 0.52 for aspect angles of 12.5 and $2.5^{\circ}$, respectively) cannot be relied upon. This is because the spectrum in the field-aligned position is insensitive to distortions from a Maxwellian form (at least for the relaxation model) and $D^{*}$ can be any value without significantly affecting the shape of the spectrum. As a result of this limitation, the suspected derived values of $D^{*}, T_{i}$ and the anisotropy (A) presented in Figure 3 have been ringed to denote that these values should be disregarded (see later discussion).

This feature of the non-Maxwellian analysis has also been noted in recent attempts to analyze CP-3 data (over a range of aspect angles) where the electric fields were not as large as to produce non-Maxwellian effects. Any results from a non-Maxwellian analysis at aspect angles lower than 30 deg should, therefore, be treated with extreme caution, and, at least for the general ized relaxation model, there is no information on $D^{*}$ on data for a line of sight aligned with the magnetic field direction. Figure 3 a compares the average (threedimensional) ion temperature derived from the non-Maxwellian analysis, $T_{i}$ (solid line), with the temperature derived from an analysis which assumed an isotropic Maxwellian distribution function, Tim (dashed line). Clearly, at low aspect angles, $T_{i m}$ is an underestimate of the ion temperature, whereas $\mathrm{T}_{\text {im }}$ overestimates $\mathrm{T}_{i}$ at large aspect angles. In contrast, Figure $3 d$ shows $\mathrm{T}_{i}$ compared with the line of sight temperature, $\mathrm{T}_{\mathrm{f}}$ derived from the non-Maxwellian fits (dashed line) and Maxwellian fits $\mathrm{T}_{\mathrm{fm}}$ (dotted line). Note that numerically $\mathrm{T}_{\mathrm{fm}}=\mathrm{T}_{\mathrm{im}}$; the difference between $\mathrm{T}_{\text {im }}$ and $\mathrm{T}_{\mathrm{fm}}$ is one of definition: $\mathrm{T}_{\mathrm{im}}$ is a three-dimensional temperature assuming an (isotropic) Maxwellian, whereas $\mathrm{T}_{\mathrm{fm}}$ is a onedimensional temperature which assumes a biMaxwellian distribution function. Tf is greater than $T_{i}$ for $\psi>54.7 \mathrm{deg}$ and less than $T_{i}$ for lower aspect angles. Figure 3 d shows that at all but one aspect angle the distortion of the line of sight ion velocity distribution from a Maxwellian gives $\mathrm{T}_{\mathrm{fm}}$, which does not in general equal the real line of sight temperature, $\mathrm{T}_{\mathrm{f}}$, in fact $\mathrm{T}_{\mathrm{fm}}>\mathrm{T}_{\mathrm{f}}$.

The equal values of $\mathrm{T}_{j}$ and $\mathrm{T}_{\mathrm{f}}$ at $\Psi=54.7 \mathrm{deg}$ are the natural consequence of using onedimensional ion velocity distributions at different aspect angles which are all correctly normalized and consistent with the one threedimensional ion velocity distribution function, as is the case for the Suvanto [1988] routine used here and in the Raman et al. [1981] algorithm on which it is based. This is because for any gyrotropic distribution function, $\mathrm{T}_{\mathrm{f}}$ will equal $\mathrm{T}_{i}$ at $\Psi=54.7 \mathrm{deg}$. However, the one- 
dimensional line of sight ion temperature estimate $\mathrm{T}_{\mathrm{im}}$ does not equal the $\mathrm{T}_{i}$ value at $\Psi=$ $54.7^{\circ}$ but does near $44^{\circ}$ (see Figure 3a.) Note that the three-dimensional ion temperature estimate, $\mathbb{T}_{i}$, and the temperature anisotropy, A, are derived from line of sight spectra, using the fitted $D^{*}$ with the assumption that the ion velocity distribution function is described by the Raman et al. [1981] form with $T_{\| 1}$ $=\mathrm{T}^{*}$. This assumption appears to be at least partially vindicated by the success of this distribution function in predicting the aspect angle dependence of the observed spectra [Winser et al:, 1987]. The line of sight temperatures $\left(\mathrm{T}_{\mathrm{f}}\right)$, derived do not depend to any great extent on the distribution function form adopted (Suvanto et al., submitted, 1988), and, from Figure $3 \mathrm{~d}$ and (2) (with the assumption that $\mathrm{T}_{\|}$ and $T \perp$ are constant over the scan) the aspect angle dependence of $\mathrm{T}_{\mathrm{f}}$ gives roughly the same anisotropy $(\sim 2)$ as the individual data points in the second panel, for the range of scan positions ( $30<\Psi<60 \mathrm{deg}$ ) for which the ion drift was large but where $\Psi$ was sufficient to allow determination of $D^{*}$. Hence this too indicates that the Raman et al. [1981] ion velocity distribution is adequately representing the ion velocity distribution function, at least to first order, and is certainly much better than an isotropic Maxwellian. This is an important finding because the only previous experimental verification of this distribution function came from satellite RPA data which only looked at the field-perpendicular velocity distribution [StMaurice et al., 1976]. Recent Monte-Carlo simulations have indicated that more complex distribution functions may be required, giving parallel ion temperatures which are not equal to $\mathrm{T}^{*}$ and even distortion of the parallel ion velocity distribution from a Maxwellian (J.-P. St-Maurice, private conmunication, 1988). However, from these observations we can say that these must be second-order effects, and introducing $D^{\star}$ with the simple Raman et al. distribution function produces a largely consistent set of temperatures.

\section{Effects Of Mixed Composition On The Fitting Procedure}

In addition to increases in both the ion temperature and $D^{\star}$ due to enhancements in auroral electric fields, the local ion composition may also change. The heating which results from the large electric fields will cause an upwelling of the neutral atmosphere, providing enriched concentrations of $\mathrm{O}$ and $\mathrm{N}$ in the $\mathrm{F}$ region (see, for example, Iathuillere [1987] and references therein). In addition, the rates of the ion-atom interchange reactions between $\mathrm{O}^{+}$and $\mathrm{N}$ or NO are increased under conditions of large electric fields, and hence $\mathrm{NO}^{+}$can exist in abundance at the EISCAT tristatic intersection height of 275 $\mathrm{km}$. The presence of such ions will also influence the interpretation of the observed spectra. This point is illustrated more clearly by the synthesized spectra presented in Figure 4. This shows three spectra for a plasma with electron density of $4 \times 10^{11} \mathrm{~m}^{-3}$, electron temperature equal to the temperature of both ion species $\left(\mathrm{T}_{\mathrm{e}}\right.$ $=\mathrm{TO}^{+}=\mathrm{T}_{\mathrm{NO}}{ }^{+}=2000 \mathrm{~K}$ ) and $\mathrm{D}^{*}$ for both species of 1.3 , with a fractional composition of $\mathrm{O}^{+}$ions,

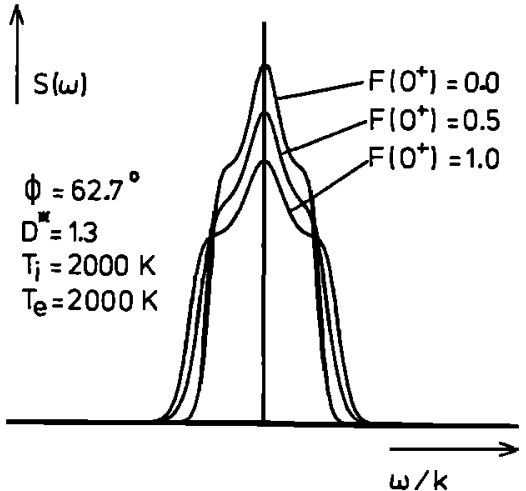

Fig. 4. Synthesized spectra for a fixed look direction but with different proportions of $0^{+}$ ions present. The fractional composition of atomic ions, $F\left(O^{+}\right)$, varies between 0.0 and 1.0 . In all cases, $D^{*}$ for both ion species were equal, as were the ion and electron temperatures.

$\mathrm{F}\left(\mathrm{O}^{+}\right)$, of $1.0,0.5$, and 0.0 , respectively. The spectral shape for a pure $0^{*}$ plasma and that for a pure $\mathrm{NO}^{+}$plasma are very similar, in fact, the fitted $D^{*}$ values for $F\left(O^{+}\right)=1.0$ and $F\left(\mathrm{NO}^{+}\right)=1.0$ under the same temperature and electron density conditions should be the same. However, the spectral power densities for the pure $\mathrm{NO}^{+}$case are much higher, and the spectrum is much narrower. On the other hand, for $F\left(O^{+}\right)=0.5$ (equal proportions of $\mathrm{O}^{+}$and $\mathrm{NO}^{+}$) the spectral shape is very different. In general, there is, of course, no reason to expect that $D^{*}$ for $0^{+}$and $\mathrm{NO}^{+}$ions in a mixed composition plasma should be the same [St-Maurice et al., 1976].

The analysis procedure described in section 3 was modified so that it had the capability of fitting $D^{*}$ for two separate ion species, with the assumption that the temperatures of both ions were the same. This assumption can clearly be questioned and may introduce errors in the derived values of $D^{*}$. However, the true relationship between the temperatures of different ion species under conditions prevailing during the time these measurements were made is unknown, and assuming they are equal is a reasonable first approximation. This problem clearly represents an area for further study. To simplify the analysis, the ion composition was assumed and not fitted, and fitting was carried out for a range of composition values. The analysis was performed on the integrated spectrum observed at the intersection height in position 5 in the EISCAT scan for different composition models ranging from $F\left(O^{+}\right)=0$ to $F\left(0^{*}\right)=1$. This spectrum was chosen because it was very symmetrical and the individual spectra which were averaged to produce it showed exactly the same features. The results of the analysis are presented in Figure 5. Clearly, the derived plasma temperatures are at a minimum when a pure $O^{+}$plasma is assumed. These increase steadily as the proportion of molecular ions is increased, with differences of nearly $2000 \mathrm{~K}$ in electron temperature and $1300 \mathrm{~K}$ in ion temperature arising between a pure $\mathrm{O}^{+}$and a pure $\mathrm{NO}^{+}$plasma (top panel). Reality is probably somewhere between 


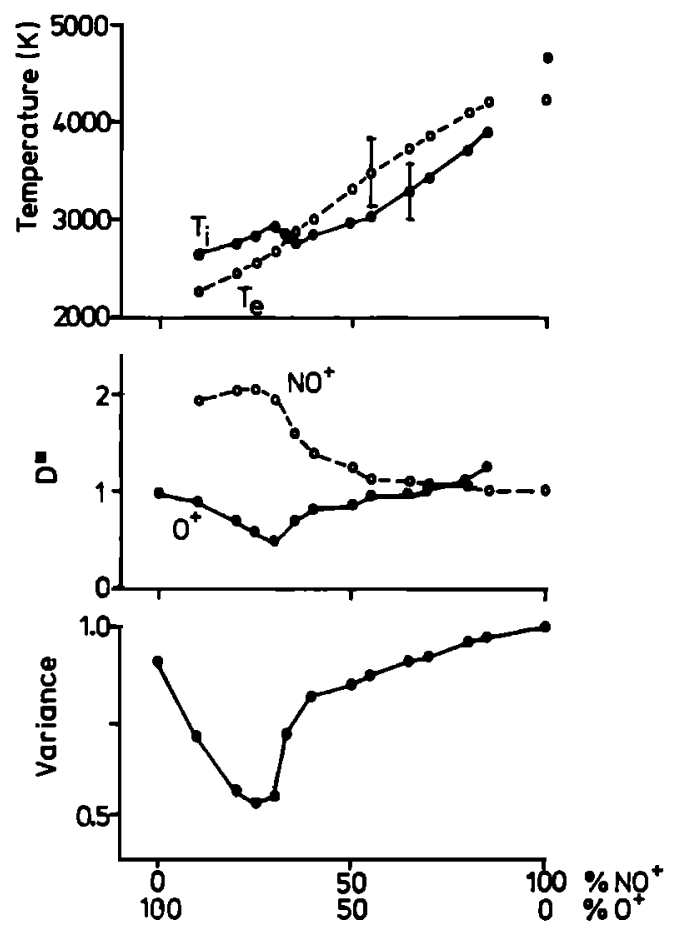

Fig. 5. Fitted values of (a) ion and electron temperature and (b) $D^{*}$ for both atomic and molecular ions as a function of ion composition. These results were obtained from an analysis of the integrated spectrum obtained at $275 \mathrm{~km}$ in scan position 5 of the EISCAT CP-3-E experiment on August 27 (refer to Figure 1). The bottom panel shows the fit variance (arbitrarily normalized to unity at its maximum value) as a function of composition. The integration period was $110 \mathrm{~s}$, and the aspect angle remained fixed at $62.7^{\circ}$. The error bars represent the maximum uncertainty obtained in the derived parameters.

these extremes, and the bottom panel may well be a good indication of what this is. It illustrates the fit variance as a function of composition, showing a very well defined minimum at about $F\left(0^{+}\right)=0.75$. The fact that the derived temperatures show no strange behavior at the minimum in variance testifies to its reliability. Note that the minimum fit variance is roughly half that for either pure $\mathrm{O}^{+}$or pure $\mathrm{NO}^{+}$plasma. The middle panel of Figure 5 shows the fitted values of $D^{*}$ for both species as a function of composition. As with the derived temperatures, there are no discontinuities or anomalous features at the composition where the minimum variance occurs. However, for the best fit, $D^{*}$ for $\mathrm{NO}^{+}$is significantly higher than that for $\mathrm{O}^{+}$. In fact, the plasma must be more than $75 \%$ NO ${ }^{+}$for this not to be the case.

A similar non-Maxwellian analysis was attempted on the gate immediately above the intersection height (centered at $295 \mathrm{~km}$ ), and the results are presented in Figure 6 . The general behavior is very similar to that presented in Figure 5. There are, however, some important differences. First, the minimum in fit variance (although unique) is not as pronounced as that in
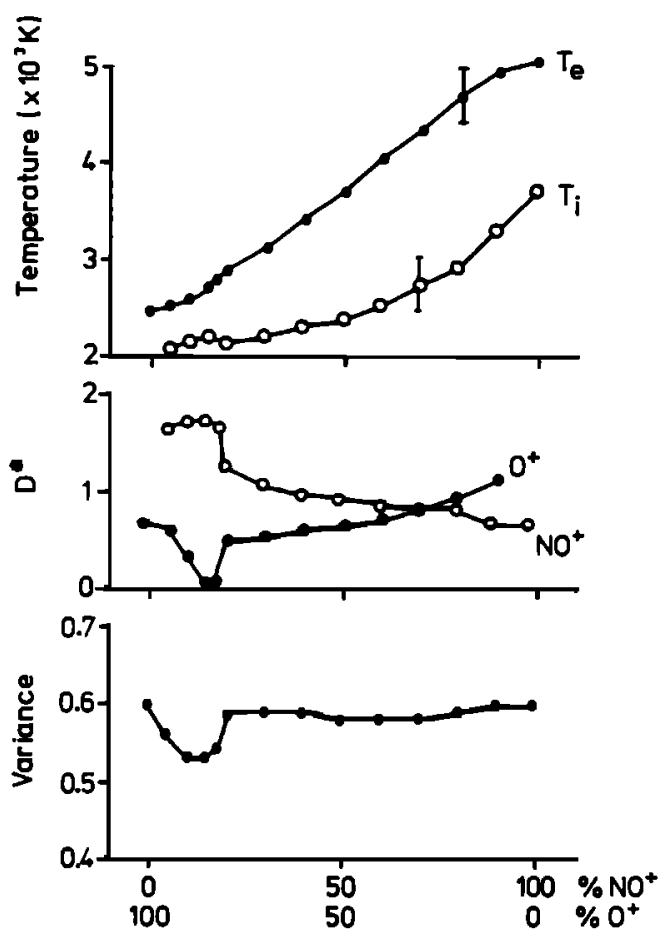

Fig. 6. As Figure 5, but at an altitude of 295 $\mathrm{km}$.

Figure 5. Second, it occurs at $F\left(0^{+}\right)=0.85$, which is consistent with a reduction in the molecular ion content with increasing altitude. The derived values of $D^{*}$ for $\mathrm{NO}^{+}$, al though lower than the corresponding values shown in Figure 5 , are still significantly larger than those for $0^{+}$ (for $\mathrm{F}\left(\mathrm{NO}^{+}\right)<0.7$ ), which reduce to almost zero at the minimum variance.

Figure 7 shows the electron temperature profile observed while the antenna was in position 4 of the scan cycle, that is, the scan position immediately prior to that described above. Reference to Figure 2 of Winser et al. [1987] shows that the measured electric fields were not unusually large in this position. It is expected that the enhanced electric fields observed in position 5 of the scan would have little effect on the measured electron temperature profile (i.e., the electron temperature deduced assuming a Maxwellian distribution function $\left.\left(T_{e m}\right)=T_{e}\right)$. It might therefore be expected that the electron temperature profile in this position should be very similar to that observed in position 4 . Thie points denoted by the open circles and marked "a" and "b" on Figure 7 represent the electron temperatures derived from the non-Maxwellian analysis of the data (at position 5) which gave the minimum variances as presented in Figures 5 and 6 . The horizontal lines represent the range of temperatures derived for a composition which varied between $F\left(O^{+}\right)=0.0$ and 1.0. Clearly the "minimum variance" points lie very close to the profile observed in the previous position, indicating that these fits are more reliable that those for different compositions.

The analysis procedure was repeated for the two gates immediately below the intersection 


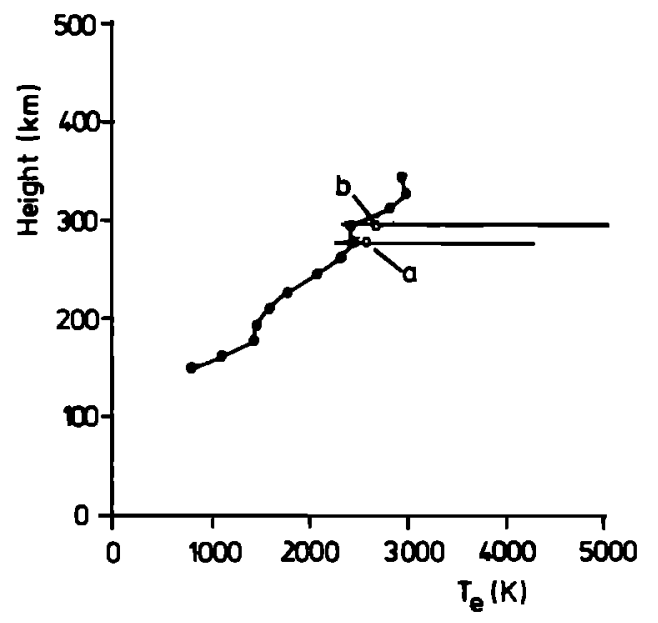

Fig. 7. Integrated electron temperature profile measured while the Tromso antenna remained stationary in position 4 of the CP-3-E scan period 1300 to 1330 UT. The points marked "a" and "b" on the plot are the electron temperatures obtained at the minimum variances presented in Figures 5 and 6 , respectively. The error bars on these two points represent the range of temperatures derived from the nonMaxwellian analysis for $\mathrm{F}\left(\mathrm{O}^{+}\right)$varying between 0.0 and 1.0 .

height at position 5 . These were centered at 256 and $237 \mathrm{~km}$. As before, $\mathrm{T}_{\mathrm{e}}$ and $\mathrm{T}_{\mathrm{i}}$ were minimum for $\mathrm{F}\left(\mathrm{O}^{+}\right)=1.0$ and maximum for $\mathrm{F}\left(\mathrm{NO}^{+}\right)=1.0$. In both cases the fit variance exhibited very little change with varying composition; however, the electron temperatures (for both heights) which were closest to the temperature profile presented in Figure 7 occurred when $\mathrm{F}\left(\mathrm{O}^{+}\right)=0.7$ and 0.58 , respectively. The analysis also revealed that $D^{*}$ for $\mathrm{O}^{+}$became larger than that for molecular ions at lower al titudes.

Some of the features of the fits, for example the sudden variations of $D^{*}$ in Figure 6 , imply that there could be two sets of solutions, and discontinuities can occur when the fitting procedure starts to iterate to a minimum of a different "valley" in the variance surface. In this paper we do not attempt a survey of the forms of these variance surfaces. However, the results shown here were obtained by iterating from 20 values for the initial $D^{*}$ (between 0 and $2)$, and in no cases did this cause a convergence to another solution (i.e., along a different valley of the surface). This was always true except at small aspect angles $\left(\Psi<20^{6}\right)$, where the derived values ranged from near zero to about 3.0. This in turn will result in erroneous threedimensional ion temperature estimates, $\mathrm{T}_{i}$, for
observations in (or close to) the field-aligned direction. This point is demonstrated by (3) and (6): The analysis programe will match the lineof-sight temperature, $T_{f}$, but if $D^{*}$ is in error so will be $T_{i}$. This behavior and its causes have been discussed by Suvanto et al. [submitted, 1988]: Basically, at low aspect angles, large changes in $D^{\star}$ produce very small changes in the spectra, and these can easily be lost in random spectral noise and $D^{*}$ cannot be determined. This is why the ringed points in Figure 3 must be disregarded.

\subsection{Relevance of $D^{\star}$ for Mixed Species}

The above analysis revealed that at altitudes greater than about $260 \mathrm{~km}$ the values of $D^{*}$ derived for molecular ions were significantly larger than those values derived for pure $0^{+}$ ions. This differs from the experimental results of St-Maurice et a]. [1976], who found that $D^{*}$ for $\mathrm{NO}^{+}$was only a fraction of that for $\mathrm{O}^{+}$. This seems a more likely situation, since charge exchange is a very inefficient production mechanism for $\mathrm{NO}^{+}$at $\mathrm{F}$ region heights.

Photoionization and charge exchange between $0^{+}$ $\left({ }^{2} \mathrm{D}\right.$ and ${ }^{2} \mathrm{P}$ states) and $\mathrm{N}$ are, however, major production mechanisms for $\mathrm{N}^{+}$, , with the latter dominating above about $270 \mathrm{~km}$ ' [Fox and Dalgarno, 1985]. The chemical lifetime of $\mathrm{N}_{2}^{+}$in the upper F region is about $18 \mathrm{~s}$, which is considerably larger than the corresponding ion gyroperiod. It is therefore likely that when $\mathrm{N}^{+}$is produced in the presence of large electric fields, the distribution function will rapidly become toroidal. The $D^{*}$ observations could therefore be explained if $\mathrm{N}^{+}$formed a significant proportion of the molecular ion content during the observations presented here. However, what is not understood is why the $\mathrm{N}^{+}$ions should occur in a narrow layer centered on about $280 \mathrm{~km}$ altitude.

Hoffman et al. [1974] presented daytime observations from the ISIS 2 satellite which showed that under storm conditions the concentration of $\mathrm{N}^{+}$was as high as $10 \%$ of the $0^{+}$ concentration at ah altitude of $1400 \mathrm{~km}$. Brinton et al. [1978] subsequently discussed measurements from several passes of the $A E-C$ satellite which showed that similar proportions of $\mathrm{N}_{2}^{+}$exist at about $300 \mathrm{~km}$, near the latitude/time ${ }^{2}$ sector corresponding to the observations described in this paper. It is therefore not too implausible that up to $25 \%$ of the ion concentration in a narrow height range near $275 \mathrm{~km}$ could be $\mathrm{N}_{2}^{+}$, al though this would be the exception rather than the rule. The electron density was observed to drop by a factor of about 2 during the period of enhanced electric fields, probably owing to the increase in recombination rates resulting from the upwelling of the neutral atmosphere and enhancement in chemical reaction rate coefficients. This will in turn rapidly destroy $\mathrm{O}^{+}$ions in preference to molecular ions, therefore increasing the fractional composition of any $\mathrm{N}_{2}^{+}$ions that were present.

\subsection{Comparison Between Single Species and Mixed Composition Fits}

Finally, we consider the effects of introducing a mixed ion composition into the routines which calculate the non-Maxwellian distribution functions, which are used to construct the synthesized spectrum for comparison with the observations. Consider in particular the spectrum observed at the intersection height in position 5 of the scan. Reference to Figure 1 illustrates that the simulated spectrum which assumes a single $\left(0^{+}\right)$ion species is qualitatively similar to the observed spectrum. However, the well-defined shoulders (a characteristic feature of non-Maxellian spectra) which occur near the phase velocities of the upshifted and downshifted ion acoustic waves in the observed spectrum are not reproduced at all. 


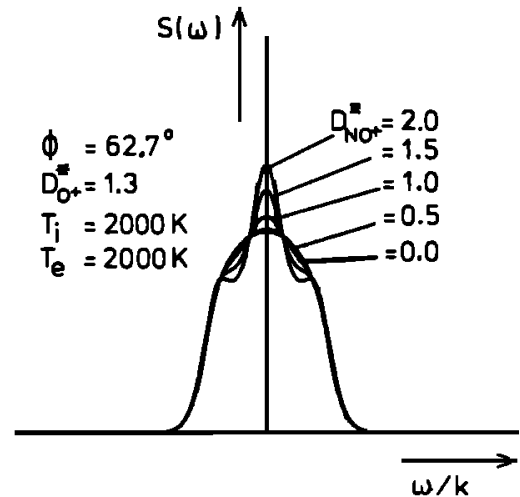

Fig. 8. Synthesized spectra for a plasma with equal proportions of atomic and molecular ions, equal ion and electron temperatures, aspect angle fixed at $62.7 \mathrm{deg}$, and $\mathrm{D}^{*}$ for $\mathrm{O}^{+}$ions fixed at 1.3 but $D^{\star}$ for molecular ions varying between 0.0 and 2.0 .

There is a very subtle tradeof $f$ between enhancing the shoulders and changing the slope of the sides of the spectrum. In the single species case it is not possible to improve one without (adversely) affecting the other.

Figure 8 shows the effects on the spectrum of mixed composition $\left(\mathrm{F}\left(\mathrm{O}^{+}\right)=\mathrm{F}\left(\mathrm{NO}^{+}\right)=0.5\right)$ with fixed $D^{*}$ for $0^{+}$ions $(=1.3)$ and varying $D^{*}$ for molecular ions. This also allows $D^{*}$ for molecular ions to be greater than that for atomic ions, which was suggested in the data. For $\mathrm{D}_{\mathrm{NO}}^{*}=0.0$ the spectrum is very similar to the single species simulation shown in Figure 1. It is also very difficult to distinguish this spectrum from a Maxwellian spectrum characteristic of high ion temperature. As $D^{*} O^{+}$is increased, the central peak becomes much sharper, and "shoulders" start to form, but without much change in the slopes of the spectrum wings. Once $\mathrm{DNO}^{*}+$ comes close to or exceeds $D_{0}^{*}$, the changes in the spectrum are very much more enhanced than they are for low $\mathrm{D}_{\mathrm{NO}}^{*}+$. This means that the spectral shape is not very sensitive to composition when the molecular ion distribution is much less distorted from a Maxwellian than the $\mathrm{O}^{+}$ion distribution. However, when the molecular ion distribution is equally as distorted as or more distorted than that of the atomic ions, the spectral shape is very sensitive to the composition, which is why such "good fits" were obtained in Figures 5 and 6, allowing estimates of the fractional composition to be made. M. Iockwood et al. (Incoherent scatter radar observations of non-Maxwellian ion velocity distributions in the auroral F region, submitted to Journal of Geophysical Research, 1988) have found that performing a similar analysis to that described above on spectra obtained with the UKPOIAR experiment provides no useful information on composition. This would suggest either that $D^{*}$ was less for molecular species than for $\mathrm{O}^{+}$ions and hence that $\mathrm{NO}^{+}$was the dominant molecular ion during the periods when their measurements were made, or that the molecular ion density was too small to significantly affect the spectrum shape regardless of which molecular species were present.

At this point, it is worth looking at how the introduction of mixed composition affected the

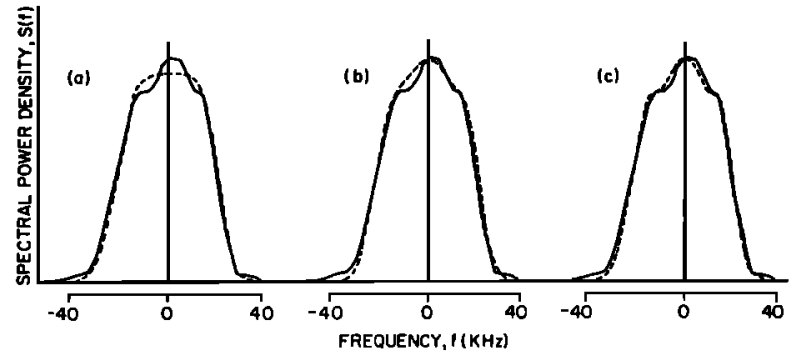

Fig. 9. The integrated incoherent scatter spectrum observed at position 5 in the scan, superimposed on (a) the best fit Maxwellian spectrum obtained with the standard analysis method, (b) the best fit spectrum obtained with a single-species, non-Maxwellian analysis, and (c) the best fit spectrum obtained with a two-ion non-Maxwellian analysis.

spectrum fitted to that observed at the intersection height in scan position 5. Figure 9 shows the observed spectrum and the best fits for a Maswellian distribution function, a single species non-Maxwellian distribution function, and a mixed composition non-Maxwellian distribution function. As mentioned before, the single species best fit is a compromise between reproducing the ratio of the central peak to the ion acoustic shoulders and the slope of the spectrum sides, and, al though it reproduces the general shape quite well, it cannot mimic the detailed shape near the peak. The mixed composition spectrum, on the other hand, produces a much more favorable comparison with the observations. This also removes any ambiguity that the observed spectrum might arise from an abormally high ion temperature. Many attempts were made to simulate the observed spectrum with the assumption of a single ion species, all of which failed to produce as good a comparison as could be obtained with a mixed species composition.

\section{Conclusions}

We have briefly described a technique which will allow us to analyze incoherent scatter radar data obtained during the presence of very large auroral electric fields which can result in significant distortions of the ion velocity distribution function from a Maxwellian form. The distribution function used in this analysis was essentially that employed by Raman et al. [1981], and the spectra were calculated using the algorithm developed by Suvanto [1988].

Data were analyzed using this method for a period where the electric fields were significantly enhanced and almost constant (yielding plasma drifts of about $2 \mathrm{~km} \mathrm{~s} \mathrm{~s}^{-1}$ ) over a wide range of latitude and observing angles. A comparison of the observed and fitted spectra (assuming a pure $\mathrm{O}^{+}$ionosphere) shows that there is a good qualitative agreement between the measurements and simulations with the general features reproduced quite well. Fitted values of $D^{*}$ were generally low $(\approx 0.1)$ when the electric fields were moderately small (in the range 25 to $50 \mathrm{mV} \mathrm{m}^{-1}$ ); however, when the bulk ion drift increased above about $1 \mathrm{~km} \mathrm{~s}, \mathrm{D}^{*}$ increased to values as large as 1.3. 
A comparison was made between the ion temperatures derived from the non-Maxwellian analysis and those derived assuming a Maxwellian distribution function. What is very clear is that the latter will overestimate the true ion temperature at large aspect angles and underestimate it for smaller aspect angles. The results also show that a clear temperature anisotrojy is present in the data.

Because of the insensitivity of the incoherent scatter spectrum to distortions in the distribution function from a Maxwellian form when viewing along, or close to, the field-aligned direction, it is not advisable to use the method described above to analyze data taken at angles less than about $30^{\circ}$ to the magnetic field. Under such circumstances the non-Maxwellian analysis program would produce $D^{*}$ values which varied markedly (and apparently randomly) for different initial values of $D^{*}$. This can lead to erroneous estimates of the plasma parameters and in particular the derived three-dimensional ion temperature, $T_{i}$. This is not the case for large aspect angles: For $\Psi$ greater than about $30 \mathrm{deg}$ the non-Maxwellian analysis program produces $D^{*}$ values which are independent of the initial value arbitrarily supplied by the user.

The theoretical expressions for the nonMaxwellian distribution functions were extended to include the effects of a mixed ion composition, and the data analysis repeated with fits attempted on the electron density and temperature, ion temperature (assumed to be equal for both ion species considered), and $D^{*}$ for both $\mathrm{O}^{+}$and $\mathrm{NO}^{+}$ions. This sometimes significantly improved the analysis and indicated that the best fits (minimum variance) were obtained when the presence of molecular ions was included in the spectral synthesis routines. The proportion of molecular ions which gave the best fits reduced with increasing altitude from about $47 \%$ at $237 \mathrm{~km}$ to $15 \%$ at $295 \mathrm{~km}$.

Finally, the non-Maxwellian analysis indicated that $D^{*}$ for the molecular ions was significantly higher than that for $\mathrm{O}^{+}$ions in a restricted al ti tude region near $275 \mathrm{~km}$. This is in conflict with the experimental observations of St-Maurice et al. [1976], who showed $D^{*}$ for $\mathrm{NO}^{+}$ions to be much lower than for $0^{+}$ions. This would seem the more likely situation, as charge exchange is an inefficient method for producing $\mathrm{NO}^{+}$at the altitude of the measurements presented in this paper. However, a visual inspection of the fitted spectra confirms that a comparison between the measurements and simulations was considerably improved by including a mixed ion composition. We believe that the solution we presented is the best that could be obtained under reasonable conditions and tentatively suggest that the molecular ions present were not purely $\mathrm{NO}^{+}$but rather a mixture of $\mathrm{NO}^{+}$and $\mathrm{N}^{+}$. Charge exchange and photoionization are a $\mathrm{key}^{2}$ source of $\mathrm{N}^{+}$at $\mathrm{F}$ region heights, and the lifetime is considerably longer than the corresponding ion gyroperiod. It is therefore probable that if $\mathrm{N}^{+}$were formed, it would rapidly become toroidal. Al though $\mathrm{O}^{+}$will also become toroidal under the same conditions, it is more prone to destructive mechanisms such as the presence of polarization scatter interactions and isotropizing instabilities. The $\mathrm{N}^{+}$ions are not subject to these destructive ion-neutral interactions, as they dissociate after a lifetime of several gyroperiods, and so it is expected that $D^{*}$ for $N_{2}^{*}$ ions should be greater than $D^{*}$ for $0^{+}$.

Acknowledgments. The authors are indebted to the EISCAT Director and his staff for operating the facility and supplying the data. EISCAT is an international facility supported by the research councils of Finland (SA), France (CNRS), the Federal Republic of Germany (MAG), Norway (NAVF), Sweden (NFR) and the UK (SERC). One of us (GOINJ) was supported by the SERC during the period when the work was carried out, while another (KS) was supported by the Oskari Huttunen Foundation of Finland with assistance from an ORS scholarship and the Academy of Finland. The authors wish also to thank B.J.I. Bromage for her assistance in developing the radar data analysis programs.

The Editor thanks C. Iathuilliere and W.I. Oliver for their assistance in evaluating this paper.

\section{References}

Barakat, A. R., R. W. Schunk, and J.-P. StMaurice, Monte-Carlo calculations of the $\mathrm{O}^{+}$ velocity distributions in the auroral ionosphere, J. Geophys. Res., 88, 3237-3241, 1983.

Brinton, H. C., J. M. Grebowsky, and L. H. Brace, The high-latitude winter $\mathrm{F}$ region at $300 \mathrm{~km}$ : Thermal plasma observations from AE-C, J. Geophys. Res., 83, 4767-4776, 1978.

Fox, J. L., and A. Dalgarno, The vibrational distribution of $\mathrm{N}^{+}$in the terrestrial ionosphere, J. Geophys. Res. , 90, 7557-7567, 1985.

Hof fman, J. H., W. H. Dodson, C. R. Lippincott, and $H$. D. Hammack, Initial ion composition results from the ISIS 2 satellite, J. Geophys. Res. , 79, 4246-4251, 1974.

Hubert, D., Auroral ion distribution functions: Generalized polynomial solution of Boltzman's equation, Planet. Space Sci., 31, 119-127, 1983.

Hubert, D., Non-Maxwellian velocity distribution functions and incoherent scattering of radar waves in the auroral ionosphere, J. Atmos. Terr. Phys., 46, 601-612, 1984.

Iathuillere, C., Ion composition response to auroral energy inputs in the lower F region, Ann. Geophys., 5A, 449-454, 1987.

Iockwood, M., and T. J. Ful ler-Rowell, The modelled occurrence of non-thermal plasma in the ionospheric F region and the possible consequences for ion outflows int the magnetosphere, Geophys. Res. Lett, , 14, 371$374,1987 \mathrm{a}$.

Lockwood, M. , and T. J. Fuller-Rowell, Correction to "The modelled occurrence of non-thermal plasma in the ionospheric $\mathrm{F}$ region and the possible consequences for ion outflows into the magnetosphere," Geophys. Res. Iett., 14 , 581-582, $1987 \mathrm{~b}$.

Iockwood, M., B. J. I. Bromage, R. B. Horne, J.-P. St-Maurice, D. M. Willis, and S. W. H. Cowley, Non-Maxwellian ion velocity distributions observed using EISCAT, Geophys. Res. Lett., 14, 111-114, 1987.

Lockwood, M., K. Suvanto, J.-P. St-Maurice, K. 
Kikuchi, B. J. I. Bromage, D. M. Willis, S. R. Crothers, H. Todd, and S. W. H. Cowley, Scattered power from non-thermal $F$ region plasma observed by EISCAT - evidence for coherent echoes?, J. Atmos. Terr. Phys., 50, 467-485, 1988.

Iovhaug, U.P., and T. Fla, Ion temperature anisotropy in the auroral $\mathrm{F}$ region as measured with EISCAT, J. Atmos. Terr. Phys., 48, 959$971,1986$.

Moorcroft, D.R., and K. Schlegel, Evidence for non-Maxwellian velocity distributions in the $F$ region, J. Atmos. Terr. Phys., 50, 455-465, 1988.

Perraut, S., A. Brekke, M. Baron, and D. Hubert, EISCAT measurements of ion temperature which indicate non-isotropic ion velocity distributions, J. Atmos. Terr. Phys., 46, 531$544,1984$.

Raman, R. S. V., J.-P. St-Maurice, and R. S. B. Ong, Incoherent scattering of radar waves in the auroral ionosphere, J. Geophys. Res., 86, 4751-4762, 1981.

Schunk, R. W., and J. C. G. Walker, Ion velocity distributions in the auroral ionosphere, Planet. Space Sci., 20, 2175-2191, 1972.

St-Maurice, J.-P., and R. W. Schunk, Auroral ion velocity distributions using a relaxation model, Planet. Space Sci., 21, 1115-1130, 1973.

St-Maurice, J.-P., and R. W. Schunk, Behavior of ion velocity distributions for a simple collision model, Planet. Space. Sci., 22, 118,1974
St-Maurice, J.-P., and R. W. Schunk, Auroral ion velocity distributions for a polarization collision model, Planet. Space Sci., 25, 243$260,1977$.

St-Maurice, J.-P., and R. W. Schunk, Ion velocity distributions in the high latitude ionosphere, Rev. Geophys., 17, 99-134, 1979.

St-Maurice, J.-P., W. B. Hanson, and J. C. G. Walker, Retarding potential analyzer measurements of the effect of ion-neutral collisions on the ion velocity distribution in the auroral ionosphere, J. Geophys. Res., 81, 5438-5446, 1976

Suvanto, K. , Incoherent scattering of radar waves from nonthermal F region plasma: Analytical methods of spectral synthesis, Radio Sci., in press, 1988.

Winser, K. J., M. Iockwood, and G. O. I. Jones, Nonthermal plasma observations using EISCAT: Aspect angle dependence, Geophys. Res. Lett., 14, 957-960, 1987.

G. 0. L. Jones, University College of Wales, Aberystwyth, Dyfed SY23 3BZ, Wales. M. Lockwood and K. J. Winser, Rutherford Appleton Iaboratory, Chilton, Didcot, Oxon., OX11 OQX, England.

K. Suvanto, Blackett Laboratory, Imperial College, Iondon, SW7 2BZ, England.

(Recelved April 20, 1988;

revised July 11, 1988;

accepted July 20, 1988.) 